\title{
Investigation of the directional emissivity of materials using infrared thermography coupled with a periodic excitation
}

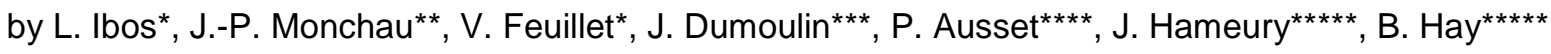 \\ *Université Paris-Est, CERTES / OSU Efluve, 61 Av. du Gal de Gaulle, 94010 Créteil, France, ibos@u-pec.fr \\ ** THEMACS Ingénierie, 23 rue Alfred Nobel, 77420 Champs-sur-Marne, France \\ *** LUNAM Université, IFSTTAR, COSYS, 44340 Bouguenais, France \\ **** Université Paris-Est, LISA / OSU Efluve, 61 Av. du G ${ }^{\text {al }}$ de Gaulle, 94010 Créteil, France \\ ${ }^{* * * * *}$ LNE, 29 Avenue Roger Hennequin, 78197 Trappes, France
}

\begin{abstract}
This article concerns the determination of the directional emissivity of materials. Several materials (conducting materials or dielectrics) are investigated and the influence of surface roughness is also considered. The experimental method used for the determination is based on the use of a periodic excitation and the recording of the surface temperature variations of the sample using infrared thermography. Several consecutive measurements are performed for emission angles varying from $0^{\circ}$ (for the determinat ion of normal emissivity) to $85^{\circ}$. The experimental device developed (SPIDER instrument) is simple compared to existing devices but the variation of directional emissivity is limited to the spectral bandwith of the camera used.
\end{abstract}

\section{Introduction}

The directional emissivity is particularly important for two applications: the correction of infrared temperature measurements for complex surfaces (varying viewing angles) on one hand and the determination of hemispherical emissivity from normal emissivity measurements (obtained from IR spectroscopy for example) on the other hand. Directional emissivity is generally nearly constant for emission angles lower than 60 degrees from the normal incidence. On the contrary, strong variations of directional emissivity are observed for angles higher than 60 degrees for both dielectric and conducting materials [1]. Variation of directional emissivity with emission angle can be deduced theoretically from the complex refractive index [2-3]. Nevertheless, theoretical relations are only valid for perfectly smooth surfaces and homogeneous materials. Differences between measurements and theory exist for rough or textured materials [4-5].

Many experimental devices were developed to determine directional emissivity of materials, for instance for glass [6-7]. Generally, these devices are quite complex and fail in measuring directional emissivity for angles greater than 80 degrees. Thus, it is still difficult to point out deviations between theoretical and experimental values in this range of emission angles. Moreover, most of the existing devices are usually based on a reflectometric method. In this case, the sample is illuminated with a directional source and the reflected flux is collected by an integrating sphere. Measuring the directional-hemispherical reflectance enables the calculation of the directional emissivity by varying the angle between the incident beam and the normal to the surface of the sample.

Current work concerns the development of a device (SPIDER instrument) allowing determining directional emissivity of dielectric or conducting materials. The method relies on the use of a periodic thermal excitation with different frequencies and the measurement of the luminance of the sample viewed from different angles. First, the experimental protocol is described, then examples of experimental results are presented and future improvements of the device are given.

\section{Experimental protocol}

\subsection{Description of the experimental device}

A schematic description and a picture of the experimental test bench are presented in figure 1 . This device is an evolution of the one presented in [8]. The investigated sample is a square thin plate $\left(44 \times 44 \mathrm{~mm}^{2}\right) \mathrm{mounted} \mathrm{on}^{2}$ Duralumin plate (same cross section, thickness $10 \mathrm{~mm}$ ) with a K-type thermocouple placed at the center. The Duralumin plate is fixed to a thermoelectric cooler (Peltier module) which applies a periodic thermal excitation. The thermoelectric cooler is fixed to a fin heat sink with fan and powered by a power amplifier controlled by an analog voltage.

A FLIR ${ }^{\odot}$ SC7300 long wave camera (spectral range 7.7-9.2 $\mu \mathrm{m}$, matrix $320 \times 256$ ) cooled by a Stirling engine is used to measure the temperature of the rear face of the sample. The acquisition of the thermal images is controlled by a digital signal [0-3.3 V] applied to the trigger input of the camera. The experimental setup is controlled by a LabView ${ }^{\odot}$ program to acquire the temperature of the Duralumin plate and to generate the control signal of the power amplifier and the camera synchronization signal. 

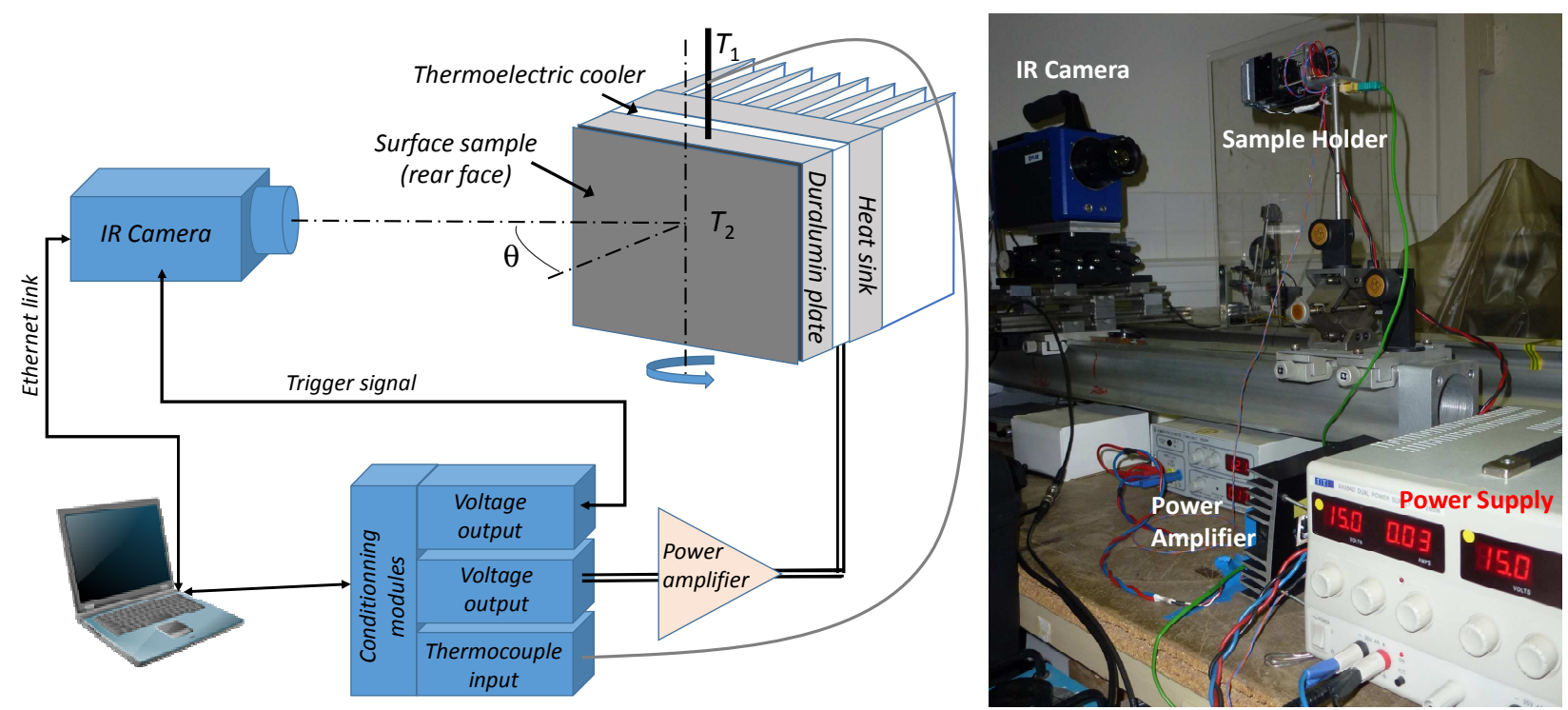

Fig. 1. Schematic description and picture of the experimental test bench (SPIDER instrument)

The thermoelectric cooler allows applying a temperature $T_{1}$ on the front face of the sample as follows:

$$
T_{1}(t)=\sum_{i=0}^{n-1} \Delta T_{1, i} \sin \left(2 \pi f_{i} t\right)=\sum_{i=0}^{n-1} \Delta T_{1, i} \sin \left(2^{i+1} \pi f_{0} t\right)
$$

The excitation signal is then a superposition of $n$ sine waves whose frequencies are successive powers of two of the fundamental frequency $f_{0}$. For the measurements, the fundamental frequency $f_{0}$ was chosen between $1 / 512 \mathrm{~Hz}$ and $1 / 128 \mathrm{~Hz}$ and the number of frequencies of the excitation signal was $n=5$. The data were acquired during nine periods of the excitation signal, that is to say a total duration ranging from $1152 \mathrm{~s}$ to $4608 \mathrm{~s}$. This duration was chosen arbitrarily. The first period is finally removed in order to consider only the measurements for which an established periodical regime is reached. The sampling frequency is $f_{\mathrm{e}}=1 \mathrm{~Hz}$. The measurement is repeated for different observation angles from $0^{\circ}$ to $85^{\circ}$, the sample holder being moun ted on a goniometer.

\subsection{Determination of the directional emissivity}

The directional emissivity is determined from the temperature amplitudes of the excitation signal and the amplitudes of the flux emitted by the surface observed at each emission angle. The temperature $T_{2}$ on the rear face of the sample (surface viewed by the camera) can be expressed as:

$$
T_{2}(t)=\sum_{i=0}^{n-1} \Delta T_{2, i} \sin \left(2^{i+1} \pi f_{0} t-\varphi_{i}\right)=\sum_{i=0}^{n-1} A_{i} \times \Delta T_{1, i} \sin \left(2^{i+1} \pi f_{0} t-\varphi_{i}\right)
$$

where $A_{i}$ is the attenuation of the temperature variation and $\varphi_{i}$ is the phase difference between $T_{1}$ and $T_{2}$ at frequency $f_{i}$. These values depend on the thermophysical properties of the sample, its thickness and the heat exchange with the environment at the rear face of the sample. The temperature of the sample surface observed by the camera can not be determined because its emissivity is unknown. Therefore the apparent temperature $T_{2}{ }^{\circ}$ of the surface is used by setting the emissivity equal to 1 :

$$
T_{2}^{\mathrm{o}}(t)=\sum_{i=0}^{n-1} \Delta T_{2, i}^{\mathrm{o}} \sin \left(2^{i+1} \pi f_{0} t-\varphi_{i}\right)=C \sum_{i=0}^{n-1} A_{i} \times \Delta T_{1, i} \sin \left(2^{i+1} \pi f_{0} t-\varphi_{i}\right)
$$

where $C$ is a coefficient depending on the emission angle and independent of the modulation frequency. The advantage of using $n$ modulation frequencies is to obtain $n$ estimates of the directional emissivity in a single experiment, the emissivity of the surface being independent of the excitation frequency. The number of frequencies is fixed to five in order to limit the temperature variation amplitudes of the sample holder.

In addition, it is recalled that the main advantage of using a modulation method is that the reflected flux from the sample surface is not required. Indeed, there is no change of the environment temperature at the modulation frequencies 


\subsection{1/qirt.2016.031}

because this temperature remains constant during the experiment. Thus, the flux collected at the modulation frequency comes only from the emission of the sample surface.

For two experiments with two different viewing angles, the orientation of the sample holder relative to the camera lens is modified. For a given angle $\theta$ given, we have:

$$
\Delta T_{2, i}^{\mathrm{o}}(\theta)=C(\theta) \times A_{i} \times \Delta T_{1, i}(\theta) \quad \forall i \in\{1 ; \ldots n\}
$$

If the sample properties do not vary from one experience to another and if the same excitation frequencies are used, the coefficients $A_{i}$ are invariant. From the measurements of temperature amplitudes on the front and rear faces of the sample at each modulation frequency, we obtain:

$$
C(\theta) \times A_{i}=\frac{\Delta T_{2, i}^{\mathrm{o}}(\theta)}{\Delta T_{1, i}(\theta)} \forall i \in\{1 ; \ldots ; n\}
$$

By normalizing to the measurement for a direction normal to the surface $(\theta=09$, we obtain:

$$
\frac{C(\theta)}{C(0)}=\frac{\frac{\Delta T_{2, i}^{\mathrm{o}}(\theta)}{\Delta T_{1, i}(\theta)}}{\frac{\Delta T_{2, i}^{\mathrm{o}}(0)}{\Delta T_{1, i}(0)}} \forall i \in\{1 ; \ldots ; n\}
$$

The ratio $C(\theta) / C(0)$ is independent of the modulation frequency and enables the calculation of the directional emissivity normalized to normal emissivity $\varepsilon(\theta) / \varepsilon(0)$ if the two measurements are made with the same camera. In practice, due to the presence of measurement noise, we obtain an estimate of the ratio $C(\theta) / C(0)$ for each modulation frequency and we must compute the average of these estimates.

The normalized directional emissivity is defined as the ratio of the luminance of the sample in the direction $\theta$ to the luminance in the direction normal to the surface. In the case of a modulation method, we will focus on the amplitude of the luminance at the different modulation frequencies $f_{i}$ :

$$
\frac{\varepsilon_{\theta}}{\varepsilon_{0}}=\frac{\tilde{L}_{\theta}\left(f_{i}\right)}{\tilde{L}_{0}\left(f_{i}\right)}
$$

The luminance measured by the camera is considered to be proportional to $T^{4}$ according to the StefanBoltzmann law [8]. Figure 2 shows the relationship between the luminance and the apparent temperature (calibration curve) provided by data extracted from Altair ${ }^{\odot}$ software for an experience performed with a highly emissive sample with $\theta=0^{\circ}$, that is to say for the most important luminanc e variations. One can observe that the chosen approximation is valid. This adjustment comes from the relationship:

$$
L=4.7712 .10^{-10} \times T^{4}-2.6178
$$

In the case where the amplitudes of temperature variation at each modulation frequency are low $\left(\mathrm{a}\right.$ few $\left.{ }^{\circ} \mathrm{C}\right)$, we obtain after simplification:

$$
\frac{\varepsilon_{\theta}}{\mathcal{E}_{0}}=\frac{\widetilde{T_{2, i}^{4}}(\theta)}{\widetilde{T_{2, i}^{4}}(0)}=\frac{\Delta T_{2, i}^{\mathrm{o}}(\theta)}{\Delta T_{2, i}^{\mathrm{o}}(0)} \times \frac{{\overline{T_{2}^{\mathrm{o}}}}^{3}(\theta)}{{\overline{T_{2}^{\mathrm{o}}}}^{3}(0)}
$$

In order to take into account variations of the excitation signal between two different experiences, the ratio of the temperature amplitudes is replaced in eq. (9) by the ratio $C(\theta) / C(0)$. So if the average temperature of the surface does not change from one experiment to another (acceptable hypothesis for the same material and the same excitation) and if the amplitudes of temperature variation are low, a simple relationship is obtained:

$$
\frac{\varepsilon_{\theta}}{\varepsilon_{0}}=\frac{C(\theta)}{C(0)}
$$




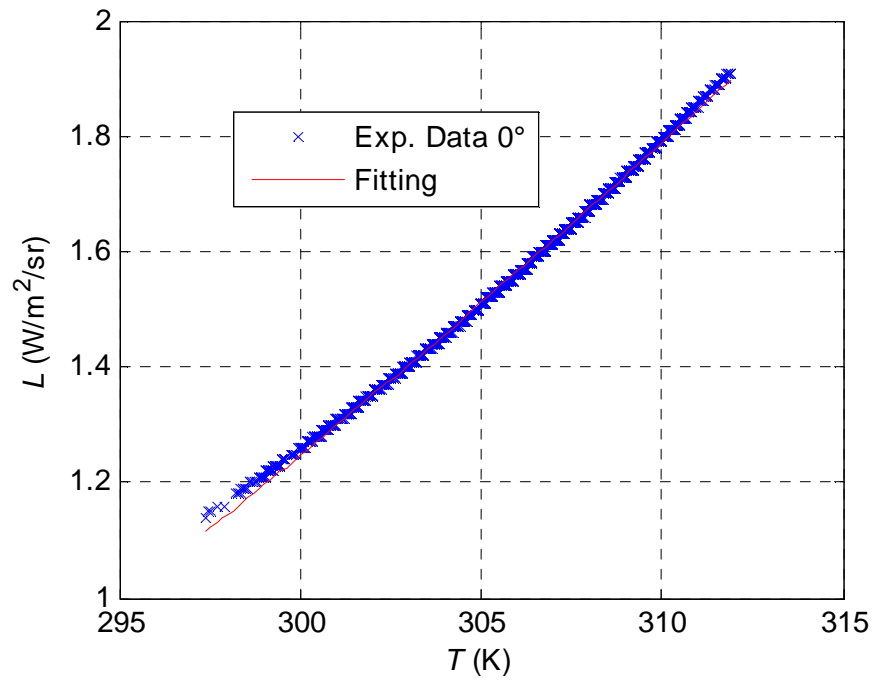

Fig. 2. Luminance measured by the camera according to apparent temperature (normal incidence to the surface)
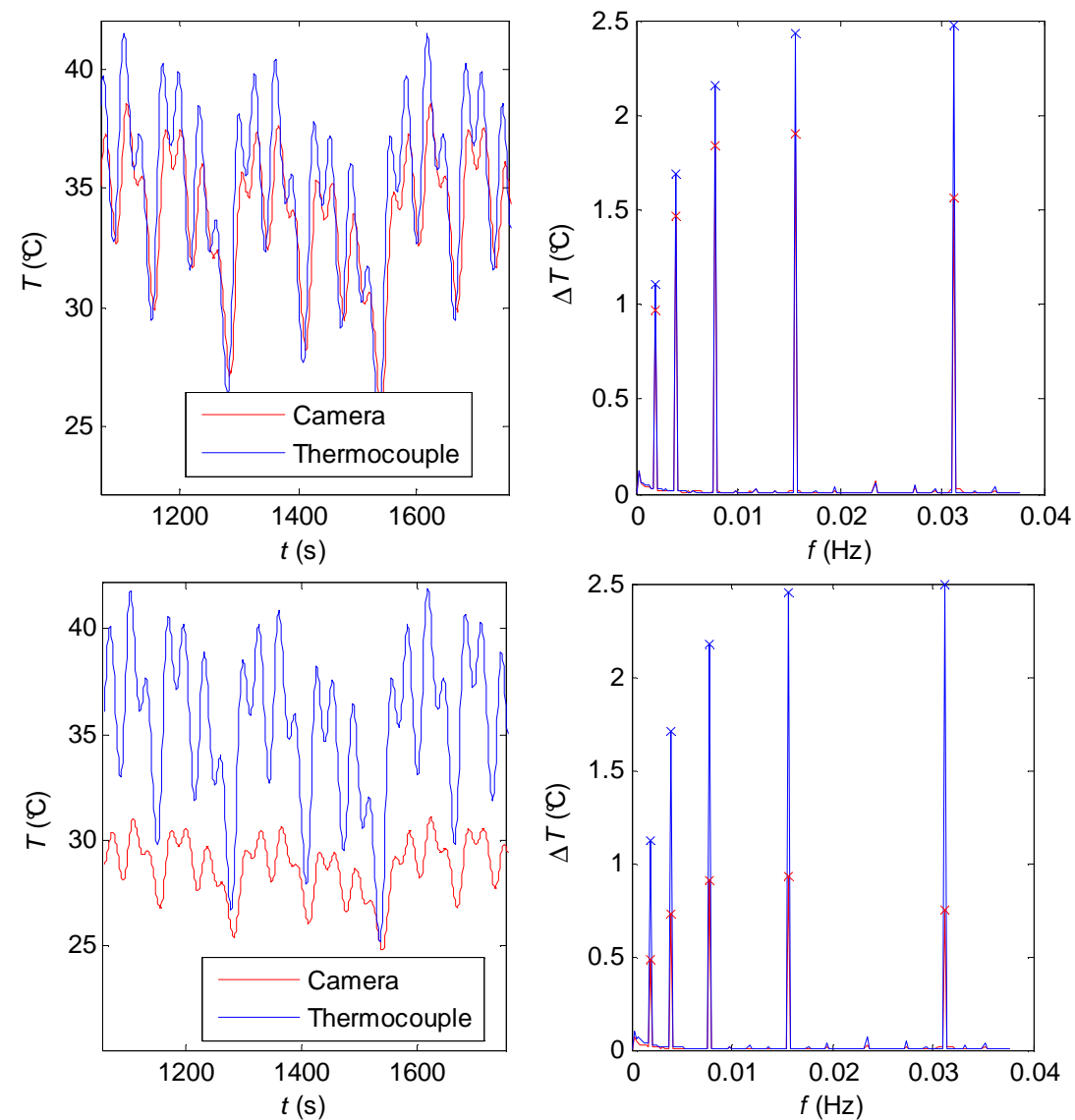

Fig. 4. Examples of measured temperatures and corresponding FFT: case of a PVC sample (up: $\theta=0$, below: $\theta=859$

\subsection{Raw signals and data processing}

Examples of thermal images for two viewing angles are shown in figure 3 . The temperature evolutions measured for $\theta=0^{\circ}$ and $\theta=85^{\circ}$ are shown in figure 4. Fast Fourier Transform (FFT) computes the amplitudes of temperature variation at the front and rear faces for each excitation frequency. The corresponding spectra are presented 
in figure 4. A much higher attenuation of temperature changes measured with the camera on the rear face of the sample is observed for grazing incidence than for normal incidence. The ratios of the temperature amplitudes between front and rear faces of the sample, that is to say the attenuation coefficients $C(\theta) \times A_{i}$, are then calculated using equation (5) for the different frequencies and emission angles $\theta$. The average and standard deviation for 5 values obtained at each emission angle are then calculated. The directional emissivities normalized to the normal emissivities are finally calculated from equations (7) to (10).

\section{Measurements}

Three materials were characterized by this method: PVC, float glass, aluminium alloy. The aluminium sample was polished prior to measurement. The surface roughness, measured using a profilometer VEECO ${ }^{\odot}$ NT-1100, allows considering these surfaces as smooth in the investigated wavelength range (see table 1). The directional emissivity could be determined up to $\theta=85^{\circ}$ for the three samples.

\subsection{Determination of the normal emissivity by a spectroscopic method}

The calculation of the directional emissivity requires to know a particular value of the emissivity, for example the normal emissivity. This parameter was determined by IR spectrometry using a Perkin-Elmer ${ }^{\odot}$ Frontier spectrometer equipped with an integrating sphere Pike ${ }^{\odot}$. A diffusing gold surface (SpectraGold ${ }^{\odot}$ ) was used as reference and its reflectance was measured by an independent method at LNE [9]. A correction of the sphere factor was applied to raw measurements to determine the directional-hemispherical reflectance. The characterized samples being opaque, normal emissivity is then calculated for each wavelength from the following relationship:

$$
\varepsilon_{\lambda}^{\prime}=1-\rho_{\lambda}^{\prime \cap}
$$

Normal spectral emissivities of the samples obtained are plotted in figure 5 . Normal emissivities in the band wavelength $\Delta \lambda$ of the camera (indicated in dotted lines in figure 5) are reported in table 1 and computed by weighting the spectral emissivity by the Planck curve for a black body at $300 \mathrm{~K}$ :

$$
\varepsilon_{0, \Delta \lambda}=\frac{\int_{\Delta \lambda} \varepsilon_{0, \lambda} L_{\lambda}^{\mathrm{o}}(T) d \lambda}{\int_{\Delta \lambda} L_{\lambda}^{\mathrm{o}}(T) d \lambda}
$$

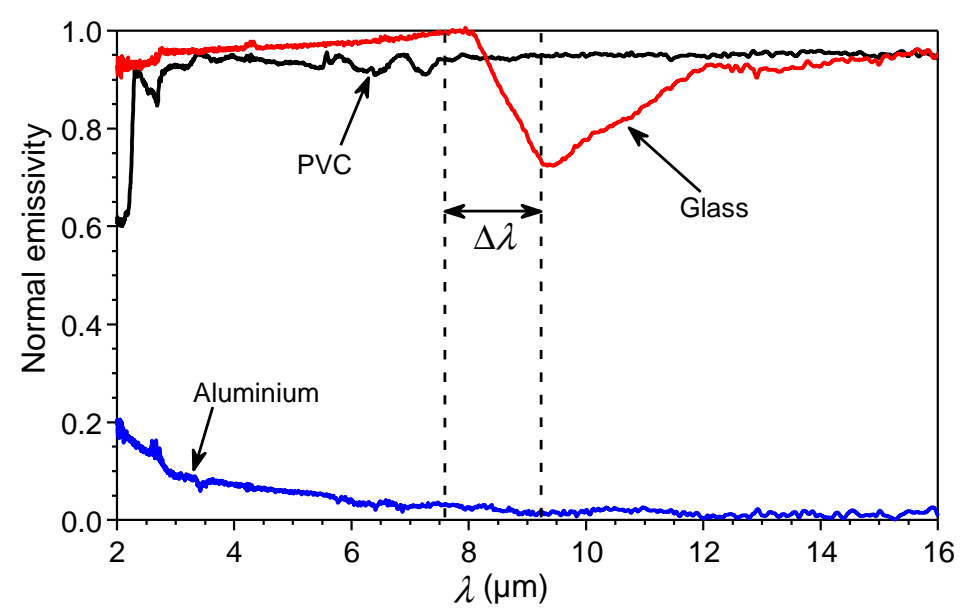

Fig. 5. Normal emissivities of the studied materials

Table 1. Properties of surfaces investigated (roughness, emissivity and refractive index)

\begin{tabular}{|l|l|l|l|l|l|}
\cline { 2 - 6 } \multicolumn{1}{c|}{\begin{tabular}{l}
$R_{\mathrm{q}}$ \\
\cline { 3 - 6 } \multicolumn{1}{c|}{}
\end{tabular}} & \multicolumn{2}{l|}{ Emissivity } & \multicolumn{2}{l|}{ Refractive index } \\
\cline { 2 - 7 } & & $\varepsilon_{0, \Delta \lambda}$ & $\varepsilon_{\Delta \lambda}$ & $n_{0}\left(\Delta n_{0}\right)$ & $\chi(\Delta \chi)$ \\
\hline PVC & & 0.95 & 0.86 & $1.47(0.14)$ & $4 \times 10^{-14} \approx 0$ \\
\hline Float glass & $<1$ & 0.90 & 0.75 & $1.05(0.11)$ & $0.68(0.06)$ \\
\hline Aluminium alloy & 1.5 & 0.025 & 0.036 & $2.92(0.52)$ & $21.2(2.2)$ \\
\hline
\end{tabular}




\subsection{Directional emissivity of the studied materials}

The directional emissivity for all investigated viewing angles is determined from the relative changes in directional emissivity and the independent measurement of the normal emissivity. Furthermore, for smooth and homogeneous materials, directional emissivity can be determined from theoretical relationships and the complex refractive index $n=n_{0}+\mathrm{j} \chi[1-2]$. In the case of a planar diopter plane, emissivities for two polarizations are defined as follows [2]:

$$
\left\{\begin{array}{l}
\varepsilon_{/ /}=1-\frac{\left(n \times \cos (\theta)-\sqrt{1-\frac{\sin (\theta)^{2}}{n^{2}}}\right) \times \overline{\left(n \times \cos (\theta)-\sqrt{1-\frac{\sin (\theta)^{2}}{n^{2}}}\right)}}{\left(n \times \cos (\theta)+\sqrt{1-\frac{\sin (\theta)^{2}}{n^{2}}}\right) \times \overline{\left(n \times \cos (\theta)+\sqrt{1-\frac{\sin (\theta)^{2}}{n^{2}}}\right)}} \\
\varepsilon_{\perp}=1-\frac{\left(\cos (\theta)-n \times \sqrt{1-\frac{\sin (\theta)^{2}}{n^{2}}}\right) \times \overline{\left(\cos (\theta)-n \times \sqrt{1-\frac{\sin (\theta)^{2}}{n^{2}}}\right)}}{\left(\cos (\theta)+n \times \sqrt{1-\frac{\sin (\theta)^{2}}{n^{2}}}\right) \times \overline{\left(\cos (\theta)+n \times \sqrt{1-\frac{\sin (\theta)^{2}}{n^{2}}}\right)}}
\end{array}\right.
$$

The directional emissivity $\varepsilon_{\theta}$ is the arithmetic mean of $\varepsilon_{/ /}$and $\varepsilon_{\perp}$ without polarization. A comparison of the measured directional emissivities with these theoretical values was performed. An estimate of the real and complex parts (respectively $n_{0}$ and $\chi$ ) of the refractive index was performed by inverse method by minimizing the sum of squared residuals between measurement and theoretical values. The values identified for each material are shown in table 1 and a comparison of experimental and theoretical data is presented in figure 6 . It is now possible from the previous results to determine the hemispherical emissivity:

$$
\varepsilon^{\cap}=\frac{\int_{0}^{\pi / 2} \mathcal{E}^{\prime}(\theta) d \theta}{\int_{0}^{\pi / 2} d \theta}
$$

The values obtained are shown in table 1. For the three investigated materials, experimental results are in accordance with theory.
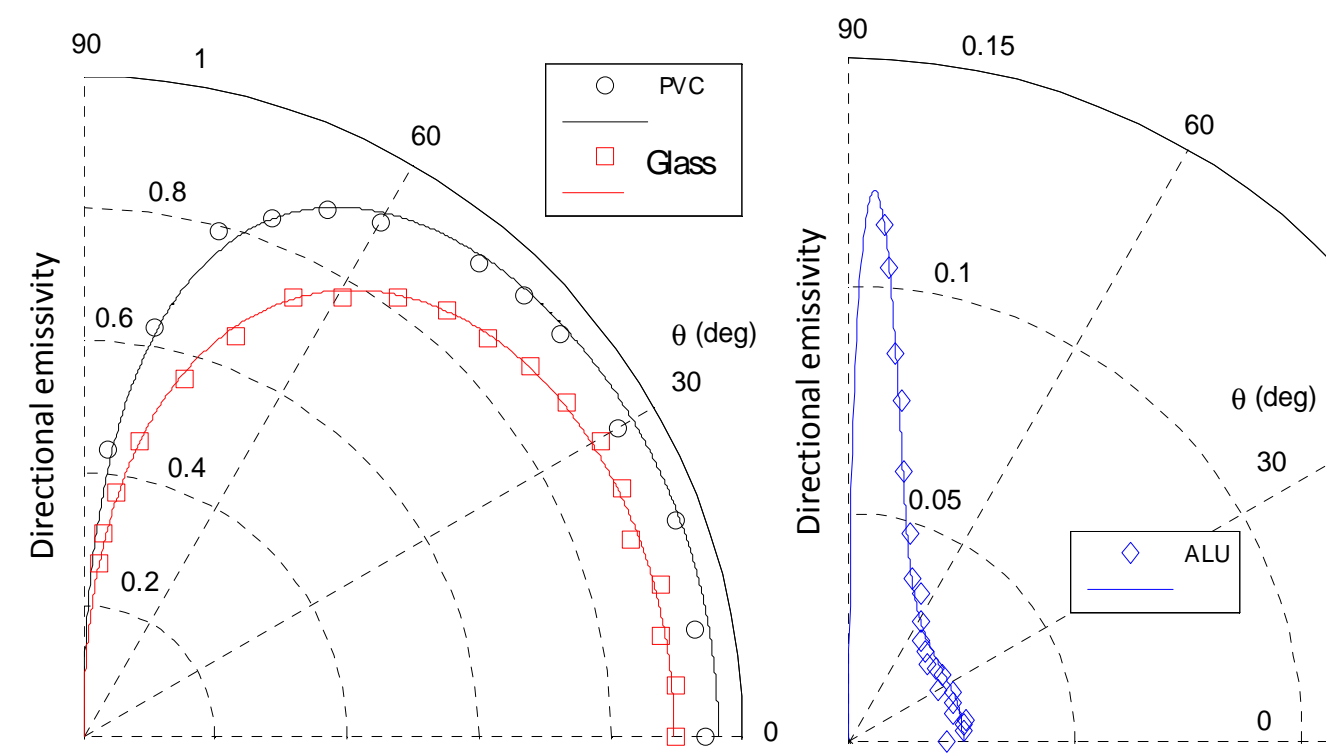

Fig. 6. Directional emissivities of the studied materials (markers refer to measurements) and comparison with theory (solid lines) 


\subsection{1/qirt.2016.031}

Results for the PVC sample show a typical behavior of a dielectric material: high emissivity almost constant up to $60^{\circ}$. A real refractive index is obtained after id entification. The corresponding dielectric permittivity (2.2) is a typical value for this material. For the glass sample, a typical behavior is also observed for this type of material [6]. Aluminium alloy behaves as a conventional metallic material. Note that it is necessary to perform measurements beyond $85^{\circ}$ to observe the significant decrease of directional emissivity for angles near grazing incidence.

\section{Conclusion and perspectives}

The use of a modulated thermal excitation avoids to measure the absolute temperature of the surface of the sample and to take into account the reflections on the surface from the environment because only the temperature and luminance changes of the surface for the excitation frequencies are considered. Thus the proposed method has the advantage to require a very simple experimental device over existing methods and can characterize both highly emissive surfaces (glass, polymers) and surfaces with low emissivity (metals). The use of a thermal camera as a detector makes it easy to work at angles close to grazing incidence. Furthermore, this also allows having the spectral emissivity to correct thermal imaging temperature fields.

However, this measurement is limited to the spectral range of the camera. The use of mono-element or multiband detectors is planned for further development of the device. Finally, only the relative variations of emissivity according to the emission angle are obtained because the surface temperature is not known. The calculation of emissivity for all angles requires additional measurement at a particular angle. In its final version, the instrument will allow measurements for emission angles between 0 and 89 degrees in different spectral bands.

\section{REFERENCES}

[1] Maldague X., Theory and practice of infrared technology for non-destructive testing, Ed. Wiley, 2001.

[2] Kauder L., Spacecraft Thermal Control Coating References, NASA-TP-2005-212792, NASA-Goddard Space Flight Center, Grenbelt, Maryland, 2005.

[3] Lorrain P., Corson D.R., Champs et ondes électromagnétiques, Armand Collin, Paris, 1979.

[4] Sacadura J.F., Influence de la rugosité sur le rayonnement thermique émis par les surfaces opaque : essai de modèle, International Journal of Heat and Mass Transfer, 15: pp. 1451-1465, 1972.

[5] Raj Koirala L., FTIR-Spectroscopic measurements of directional spectral emissivities of microstructured surfaces, Dissertation Helmut-Schmit University/University of the Federal Armed Forces, Hamburg, 2004.

[6] Geotti-Bianchini F., Total hemispherical emissivity of coated glass, European Community Report EUR 14758 EN, 1993.

[7] Janssen D., Lohrengel J., Investigation and development of a method for the measurement of the emissivity of glass, European Community Report EUR 13487 EN, 1991.

[8] Datcu S., Ibos L., Candau Y., Mattéï S., Improvement of building wall surface temperature measurements by infrared thermography, Infrared Physics \& Technology, 46: pp. 451-467, 2005. 